\title{
INFLUÊNCIA DO JEJUM SOBRE A FECUNDIDADE DE DERMANYSSUS GALLINAE (DE GEER, 1778) (ACARI, DERMANYSSIDAE)
}

\author{
E.C. Tucci' ${ }^{1}$ A.P. Prado² ${ }^{\text {, Araúlo, R.P. }}{ }^{1}$
}

${ }^{1}$ Instituto Biológico, Centro de Pesquisa e Desenvolvimento de Sanidade Animal, Av. Cons. Rodrigues Alves, 1252, CEP 04014-002, São Paulo, SP, Brasil. E-mail: tucci@biologico.sp.gov.br

\section{RESUMO}

\begin{abstract}
O trabalho teve por objetivo estudar a influência do jejum na fecundidade de Dermanyssus gallinae. Fêmeas foram colocadas em tubos para se alimentar sobre uma ave jovem e, em seguida mantidas sobjejum por períodos de 1 a 12 dias. Os resultados obtidos demonstraram que as fêmeas ovipuseram até $011^{\circ}$ dia. O número de ovos depositados variou de 1 a 8 , sendo as maiores médias obtidas nas fêmeas mantidas até o $8^{\circ}$ dia de jejum. A maior porcentagem de oviposição ocorreu até o terceiro dia de jejum, havendo uma redução do $4^{\circ}$ ao $12^{\circ}$ dia.
\end{abstract}

PALAVRAS-CHAVE: Piolhinho da galinha, desenvolvimento reprodutivo, oviposição.

\section{ABSTRACT}

THE INFLUENCE OF FASTING ON THE FERTILITY OF DERMANYSSUS GALLINAE (DE GEER, 1778) (ACARI, DERMANYSSIDAE). The objective of the present study was to evaluate the influence of fasting on the fecundity of D. gallinae. Females were maintained in tubes in order to feed on a young bird and then were kept fasting for 1 to 12 days. The results demonstrated that females laid eggs up to the 11th day. The number of eggs laid ranged from 1 to 8 , and the greatest means were obtained in females kept up to 8 days fasting. The greatest percentage of eggs laid occurred up to the 3 rd day of fasting, with a decrease from the 4 th to the 12 th day.

KEY WORDS: Poultry mites, development, reproduction, oviposition.

\section{INTRODUÇÃO}

Dermanyssus gallinae é um dos principais ectoparasitas de galinhas em aviários de postura comercial. Conhecido como "ácaro vermelho", ou piolhinho das galinhas, determina sérios prejuízos à avicultura devido ao hematofagismo. (TuCC; GuIMARÃES et al., 1998). Formam suas colônias nas instalações do aviário onde permanecem a maior parte do seu ciclo, especialmente durante o dia. À noite abandonam os esconderijos à procura das aves para realizarem o repasto sangüíneo (HARRISON, 1962; Matthysse 1972; LANCASTER JUnior; Meish, 1986).

Na literatura existem poucas informações sobre os aspectos biológicos de D. gallinae e os registros disponíveis são incompletas e fragmentadas. O único estudo sobre a fecundidade deD. gallinae foi desenvolvido por Oliver Junior (1966) em observações sobre o comportamento reprodutivo desta espécie. Segundo o autor, o estímulo efetivo para a oviposição é desconhecido e diversos fatores estão associados a esta etapa do ciclo, tais como a alimentação e a cópula. Verificou que fêmeas que se alimentaram, mas não ficaram repletas de sangue, não ovipuseram, indicando que uma determinada quantidade de sangue deve ser ingerida para que a oviposição se inicie.

HARRISON (1962) constatou que esta espécie geralmente se ingurgita na primeira oportunidade e o período da hematofagia leva de 30 a 45 minutos. Com relação à quantidade de sangue ingerida, SIKES; Chamberlain (1954) verificaram que fêmeas de $D$. gallinae ingerem $0,204 \mathrm{mg}$ de sangue a cada alimentação, correspondendo a 2,7 vezes o seu peso.

Com o intuito de controlar e conhecer melhor os ectoparasitas de importância em medicina veterinária, diversos trabalhos vêm sendo desenvolvidos sobre o uso de praguicidas, hábitos alimentares, imunidade e diversidade de hospedeiros, dentre outros. Estes experimentos requerem a manutenção de colônias em laboratório e freqüentemente os aspectos biológicos do parasita são utilizados como parâmetro de avaliação.

\footnotetext{
${ }^{2}$ Universidade Estadual de Campinas, Campinas, SP, Brasil.
} 
Visando contribuir para o conhecimento da biologia desta espécie, o presente trabalho teve por objetivo estudar a influência do jejum na fecundidade de $D$. gallinae em condições de laboratório.

\section{MATERIAL E MÉTODOS}

\section{Colônia de ácaros}

As fêmeas utilizadas no presente estudo foram obtidas a partir de colônia de D. gallinae, mantida no Laboratório de Parasitologia do Centro de Pesquisa e Desenvolvimento de Sanidade Animal do Instituto Biológico de São Paulo, com ácaros provenientes de granja de postura comercial, localizada no Município

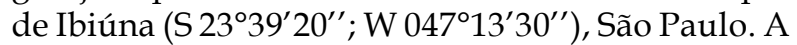
colônia foi mantida em laboratório segundo técnica descrita por Tucci (1997).

\section{Bioensaio}

A colônia de ácaros foi alimentada no início do experimento em uma avejovem e, em seguida, fêmeas ingurgitadas foram transferidas, por aspiração, para 12 pipetas de vidro, tipo Pasteur, em quantidade correspondentea $1 / 3$ de sua capacidade. Desta forma assegurou-se que todos os ácaros possuíam o mesmo estágio nutricional no início do experimento. Durante 12 dias retirou-se uma pipeta por dia para a segunda alimentação, obtendo-se ácaros submetidos a jejum por períodos de 1 a 12 dias.

Após a segunda alimentação as fêmeas ingurgitadas foram mantidas isoladas em pipetas, num total de 10 grupos com 10 repetições, perfazendo um total de 100 fêmeas/dia de jejum.

As fêmeas permaneceram sob condições controladas de umidade, $80 \% \mathrm{UR}$, temperatura de $27^{\circ} \mathrm{C}$, e fotoperíodo 12:12 (C:E) durante toda a realização do experimento.

\section{Avaliações}

As avaliações foram realizadas 7 dias após a segunda alimentação verificando-se o número de larvas e protoninfas em cada pipeta, as quais representariam o número de ovos viáveis depositados pelas fêmeas submetidas aos diferentes dias dejejum. Asleiturasforamfeitasemmicroscópioestereoscópico.

\section{Tratamento estatístico}

Os resultados obtidos analisando-se o período de jejum (variável independente) e porcentagem de fêmeas que ovipuseram (variável dependente) foram submetidos à análise de variância (ANOVA) ao teste de
Tukey para comparação de médias, ao nível global de $5 \%$ de significância.

\section{RESULTADOS E DISCUSSÃO}

Os resultados estão expressos na Tabela 1 e Figura 1.

Verificou-se que o jejum não interferiu no número de ovos postos pelas fêmeas, não havendo diferenças estatísticas significantes entre os grupos até o $9^{\circ}$ dia dejejum. As fêmeas apresentaram uma alta produção de ovos até o $8^{\circ}$ dia de jejum, com média de 4,1 a 5,4 ovos por fêmea. A partir do $9^{\circ}$ dia a média do número de ovos diminuiu de 3,2 para 2,5 ovos no $11^{\circ}$ dia.

No que se refere à fecundidade, verificou-se que houve interferência do jejum sobre fêmeas de $D$. gallinae, podendo-se distinguir 3 grupos os quais diferiram estatisticamente entre si (Fig. 1). Nos 3 primeiros dias de jejum, as fêmeas apresentaram alto índice de fecundidade (96,7\% a 99,1\%), sem diferenças estatísticas entre estes grupos e diferindo de todos os demais. Um segundo grupo, representado pelas fêmeas mantidas sob 4 e 5 dias em jejum, apresentou freqüência de oviposição média. A porcentagem de fêmeas que ovipôs neste grupo foi menor que o anterior, diminuindo para $67,1 \%$ no $4^{\circ}$ dia e $49 \%$ no $5^{\circ}$ dia. Diferenças estatísticas significantes não foram detectadas entre estes dois dias. Oterceiro grupo, representado pelas fêmeas que ficaram de 6 a 12 dias de jejum, apresentou freqüência de oviposição baixa, com a porcentagem diminuindo para $37,4 \%$ no $6^{\circ}$ dia, $23,3 \%$ no $11^{\circ}$ dia ecessando no $12^{\circ}$ dia, ondenenhuma fêmea ovipos.

Tabela 1 - Média do número de ovos e porcentagem de fêmeas de Dermanyssus gallinae que ovipuseram nos diferentes dias de jejum.

\begin{tabular}{llc}
\hline $\begin{array}{l}\text { Dias de } \\
\text { jejum }\end{array}$ & $\begin{array}{l}\text { Ovos * } \\
\text { Média } \pm \mathrm{DP}\end{array}$ & $\begin{array}{c}\text { Fêmeas que } \\
\text { ovipuseram* }\end{array}$ \\
\hline+1 & $5,37 \pm 1,41 \mathrm{a}$ & $99,1 \mathrm{a}$ \\
+2 & $5,06 \pm 1,56 \mathrm{a}$ & $99,3 \mathrm{a}$ \\
+3 & $4,82 \pm 1,51 \mathrm{a}$ & $96,7 \mathrm{a}$ \\
+4 & $5,17 \pm 1,88 \mathrm{ab}$ & $67,1 \mathrm{~b}$ \\
+5 & $5,54 \pm 1,94 \mathrm{ab}$ & $49,0 \mathrm{bc}$ \\
+6 & $4,81 \pm 1,94 \mathrm{ab}$ & $37,4 \mathrm{~cd}$ \\
+7 & $3,83 \pm 1,92 \mathrm{ab}$ & $33,3 \mathrm{~cd}$ \\
+8 & $4,26 \pm 2,62 \mathrm{abc}$ & $24,1 \mathrm{~cd}$ \\
+9 & $3,39 \pm 1,54 \mathrm{bc}$ & $24,6 \mathrm{~cd}$ \\
+10 & $1,78 \pm 0,91 \quad \mathrm{~cd}$ & $20,6 \mathrm{~d}$ \\
+11 & $2,60 \pm 1,71 \quad \mathrm{~d}$ & $23,3 \mathrm{~cd}$ \\
+12 & $0,00 \quad \mathrm{e}$ & $00.0 \mathrm{e}$ \\
\hline
\end{tabular}

*medias seguidas da mesma letra não diferem entre si ao nível de 5\% de significância (Teste de Tukey) 


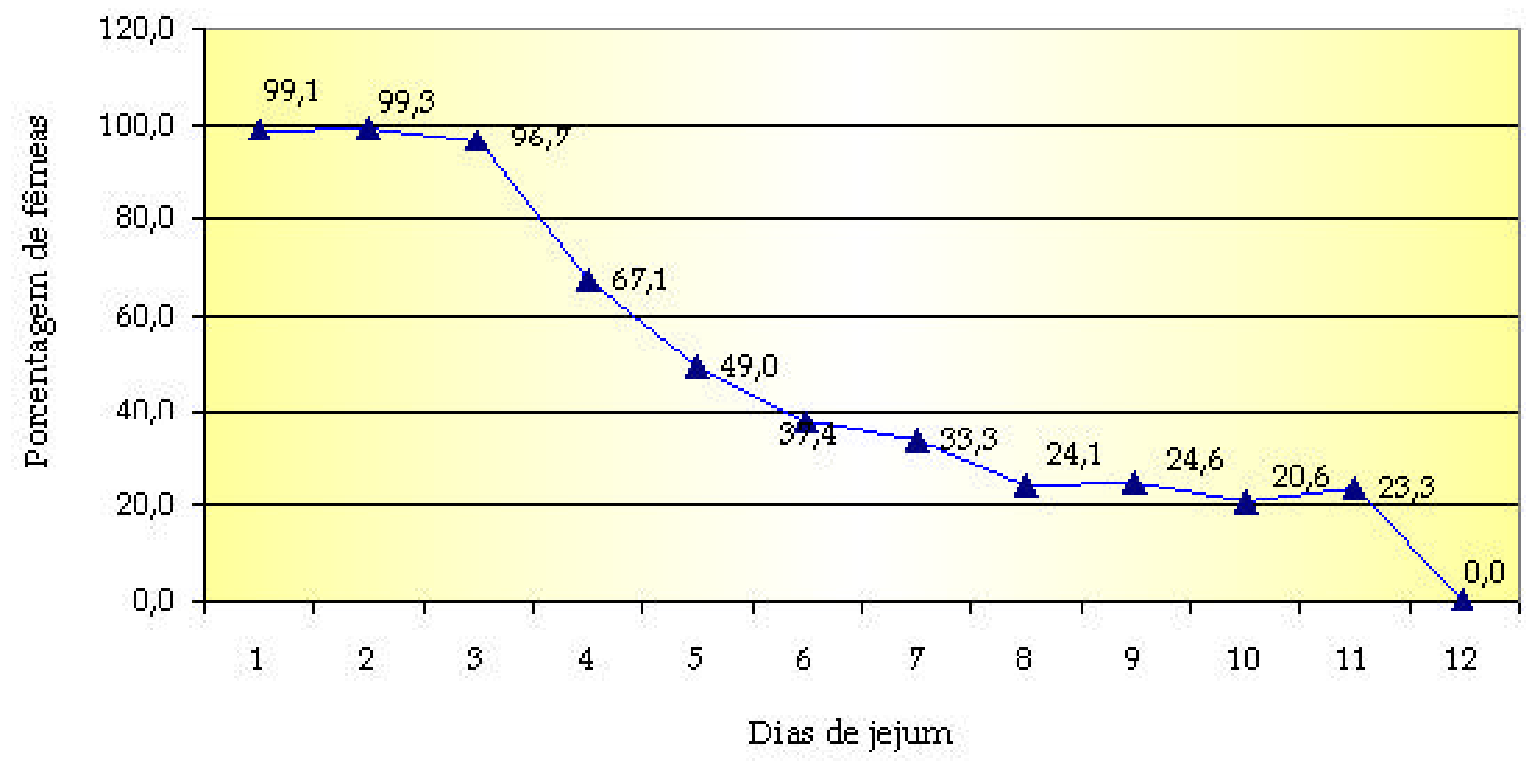

Fig. 1 - Porcentagem de fêmeas de Dermanyssus gallinaeque ovipuseram nos diferentes dias de jejum.

SiKES; CHAMBERLAin (1954) acompanharam colônias de $D$. gallinae em laboratório por 3 anos e verificaram que logo após se alimentarem as fêmeas procuram abrigos onde permanecem em colônias para depositam os ovos. Observaram que as fêmeas só começam a sair dos abrigos após um período de 3 dias, estando aptas para se alimentarem novamente assim que o hospedeiro estiver disponível.HUTCHESON;OLIVER JUNIOR (1988), acompanhando grupos de fêmeas de $D$. gallinae após a alimentação por um período de 6 dias, verificaram que a oviposição cessou após o terceiro dia, não havendo nenhum ovo no quarto dia após a alimentação. Os autores não informam a porcentagem defêmeas que ovipuseram nem o número deovos por fêmea, devido à metodologia empregada, onde as fêmeas foram mantidas em grupo. As observações realizadas por estes dois autores sugerem que em 3 dias as fêmeas estão aptas para se alimentarem novamente e isto seria o que ocorre naturalmente em condições de campo e que seria o intervalo ideal para esta espécie. No entanto, o período que as fêmeas levam para completar a oviposição não é o parâmetro mais adequado para estabelecer o intervalo entre as alimentações pelo fato da oviposição ser influenciada por outros fatores tais como temperatura e nível de ingurgitamento. Oliver JUNIOR (1966) observou que fêmeas que se alimentaram sem ficarem repletas não ovipuseram, indicando que uma determinada quantidade de sangue deve ser ingerida para que a oviposição se inicie. Tucciet al. (2008) verificaram que as fêmeas ovipõem até que todo o sangue ingerido tenha sido metabolizado e que esse período poderá variar de acordo com a temperatura. Constatou que fêmeas podem ovipor por até 9 dias sob temperatura de $15^{\circ} \mathrm{C}$ eque esse período pode cair para 48 horas sob temperatura de $25^{\circ} \mathrm{C}$. NoRDENFORs et al. (1999) constataram que a oviposição durou, em média, 28,2 dias em experimento conduzido à $5^{\circ} \mathrm{C}$ e que esse período não é influenciado pela umidade.

Os resultados obtidos no presente trabalho servirão de base para experimentos onde seja necessária a manutenção de colônias de $D$. gallinae em laboratório etambém experimentos ondea inibição da oviposição seja utilizada como parâmetro para avaliação de eficácia de substâncias acaricidas. Um exemplo disso ocorre no controle de carrapatos, onde a biologia das espécies é amplamente conhecida. Os testes para a avaliação da eficácia de substâncias carrapaticidas são padronizados na literatura internacional utilizando aspectos biológicos como parâmetro para avaliar a eficácia destes produtos, tais como o índice de produção de ovos, índice de reprodução, porcentual de eclosão, eficiência reprodutiva e porcentual de inibição de reprodução. (DruMmondet al.,1971;BENETT, 1974; DRumMOND; GLADNEY, 1978).

Os estudos desta espécie requerem a manutenção de colônias do ácaro em laboratório e o principal objetivo na manutenção de artrópodes em laboratório é o de se obter o maior número de espécimes saudáveis, de maneira prática e econômica. Desta forma, os resultados aqui obtidos contribuirão para melhorar as metodologias de manutenção de colônias de $D$. gallinae em laboratório, os quais demonstraram que, mesmo não havendo diferença entre o número de ovos postos pelas fêmeas nos diferentes dias de jejum, o intervalo de 3 dias entre as alimentações da colônia é o mais adequado por apresentar alto potencial reprodutivo (acima de 96,7\%), assegurando desta maneira o desenvolvimento ecrescimento da população em laboratório. 


\section{REFERENNCIAS}

BENNETT, G.F. Oviposition of Boophilus microplus (Canestrini) (Acarida:Ixodidae) I. Influence of ticj size on egg production. Acarologia, v.16, n.1, p.52-61, 1974.

DRUMMOND, R.O.; GLADNEY, W.J.; WHETSTONE, T.M.; ERNST, S.E. Laboratory testing of insecticides for control of the winter tick. Journal of Economic Entomology, v.64, n.3, p.686-688, 1971.

DRUMMOND, R.O.; GLADNEY, W.J. Acaricides applied to cattle for control of the Lone Star tick. Southwestern Entomologist, v.3, p.96-105, 1978.

HARRISON, I.R. The biology of poultry red mite (Dermanyssus gallinae) and its control with contact and systemic insecticides. In: INTERNATIONAL CONGRESS OF ENTOMOLOGY, 11., 1962, Vienna, Austria. Proceedings. Vienna: 1962. p.469-473.

HUTCHESON, H.J.; OLIVER JUNIOR, J.H. Spermiogenesis and reproductive biology of Dermanyssus gallinae (De Geer) (Parasitiformes: Dermanyssidae). Journal of Medical Entomology, v.25, n.5, p.321-330, 1988

KIRKWOOD, A. C. In vitro feeding of Dermanyssus gallinae. Experimental Parasitology, v.29, p.1-6, 1971.

LANCASTER, JUNIOR, J.L.; MEISH, M.V. Arthropods in livestock and poultry production. Chichester: Ellis Horwood, 1986. 305p.
MATTHYSSE, J.G. Diseases of poultry. 6.ed. Iowa: Iowa State University Press, 1972. p.815-820.

NORDENFORS H.; HOGLUND, J.; UGGLA, A. Effects of temperature and humidity on oviposition, molting, and longevity of Dermanyssus gallinae (Acari: Dermanyssidae). Journal of Medical Entomology, v.36, p.68-72, 1999

OLIVER JUNIOR, J.H Notes on reproductive behavior on Dermanyssidae. Journal of Medical Entomology, v.3, p.29-35, 1966.

SIKES, R.K.; CHAMBERLAIN, R.W. Laboratory observations on three species of bird mites. Journal of Parasitology, v.40, p.691-697, 1954.

TUCCI, E.C. A laboratory method for the rearing of Dermanyssus gallinae (DeGeer, 1778) (Acari,

Dermanyssidae). Arquivos do Instituto Biológico, São Paulo, v.64, n.1, p.1-4, 1997.

TUCCI, E.C.; GUIMARÃES, J.H. Biologia de Dermanyssus gallinae (De Geer, 1778) (Acari Dermanyssidae). Revista Brasileira de Parasitologia Veterinaria, v.7, n.1, p.27-30, 1998.

TUCCI, E. C. ; PRADO, A ; ARAUJO, R . Development of Dermanyssus gallinae (Acari: Dermanyssidae) at different temperatures. Veterinary Parasitology, v.155, p.127-132, 2008.

Recebido em 28/6/06

Aceito em 10/12/08 